\title{
Idade Avançada Reduz a Tipicidade da Apresentação Clínica em Pacientes Com Dor Torácica Aguda Relacionada a Doença Coronária Obstrutiva?
}

\author{
Does Advanced Age Reduce the Typicality of Clinical Presentation in Patients with Acute Chest Pain Related to \\ Coronary Artery Disease?
}

Pedro Henrique Correia Filgueiras, ${ }^{1}$ Antônio Maurício Cerqueira Junior, ${ }^{1}$ Gabriela Oliveira Bagano, ${ }^{1}$ Vitor Calixto de Almeida Correia, ${ }^{1}$ Fernanda Oliveira de Andrade Lopes, ${ }^{1}$ Thiago Menezes Barbosa de Souza, ${ }^{1}$ Leticia Lara Fonseca, ${ }^{1}$ Lara Queiroz Kertzman, ${ }^{1}$ Yasmin Falcon Lacerda, ${ }^{1}$ Marcia Noya Rabelo, ${ }^{2}{ }^{\oplus}$ Luis Claudio Lemos Correia ${ }^{1}$ Escola Bahiana de Medicina e Saúde Pública (EBMSP), ${ }^{1}$ Salvador, BA - Brasil

Hospital São Rafael, ${ }^{2}$ Salvador, BA - Brasil

\section{Resumo}

Fundamento: De acordo com o pensamento diagnóstico tradicional, indivíduos muito idosos estão mais predispostos a desenvolver sintomas atípicos em síndromes coronarianas agudas.

Objetivo: Testar a hipótese de que indivíduos muito idosos estão mais predispostos a manifestações de dor torácica atípica devido à doença arterial coronariana obstrutiva (DAC).

Métodos: O Registro de dor torácica inclui pacientes internados com dor torácica aguda. Primeiramente, foi construído o índice de tipicidade dessa manifestação clínica: a soma de 12 características de sintomas (8 sintomas típicos e 4 sintomas atípicos). No subgrupo de pacientes com etiologia coronariana, $o$ índice de tipicidade foi comparado entre octogenários e não octogenários. A significância estatística foi definida por $\mathbf{p}<0,05$.

Resultados: 958 pacientes foram incluídos no registro, sendo que 486 (51\%) tinham etiologia supostamente coronariana. Nesse grupo, $59(12 \%)$ octogenários (idade $84 \pm 3,5 ; 50 \%$ homens) foram comparados a 427 pacientes com idade $<80$ (60 \pm 12 anos; $71 \%$ homens). $O$ índice de tipicidade em octogenários foi $3,42 \pm 1,92$, que é semelhante ao de não octogenários $(3,44 \pm 1,74 ; p=0,092$ na análise univariada e $p=0,80$ após ajuste para sexo pela análise de variância ANOVA). Também não houve diferença estatisticamente significativa quando a amostra foi dividida em idade mediana ( 62 anos; $3,41 \pm 1,77$ vs. $3,49 \pm 1,77 ; p=0,61$ ). Não houve associação linear estatisticamente significativa entre idade e índice de tipicidade $(r=-0,05 ; p=0,24)$. A análise de regressão logística para predição de DAC na amostra geral de 958 pacientes não mostrou interação do índice de tipicidade com a idade numérica $(p=0,94)$, octogenários $(p=0,22)$ ou idade acima da mediana $(p=0,74)$.

Conclusão: Em pacientes com dor torácica aguda de etiologia coronariana, a idade avançada não influencia o quadro clínico típico.

Palavras-chave: Idoso; Dor no Peito; Síndrome Coronariana Aguda; Prognóstico.

\begin{abstract}
Background: According to traditional diagnosis thinking, very elderly individuals are more predisposed to develop atypical symptoms in acute coronary syndromes.

Objective: To test the hypothesis that very elderly individuals are more predisposed to atypical chest pain manifestations due to obstructive coronary artery disease (CAD).

Methods: The Registry of Thoracic Pain includes patients admitted with acute chest pain. Firstly, the typicality index of this clinical manifestation was constructed: the sum of 12 symptom characteristics (8 typical and 4 atypical symptoms). In the subgroup of patients with coronary etiology, the typicality index was compared between octogenarian and non-octogenarian individuals. Statistical significance was defined by $p<0.05$.

Results: 958 patients were included in the registry, and 486 (51\%) had a supposedly coronary etiology. In this group, 59 (12\%) octogenarians (age $84 \pm 3.5,50 \%$ men) were compared to 427 patients aged $<80$ (60 12 years, $71 \%$ men). The typicality index in octogenarians was
\end{abstract}

Correspondência: Luis C. L. Correia •

Av. Princesa Leopoldina 19/402. CEP 40150-080, Salvador, BA - Brasil

Email: luisclcorreia@gmail.com

Artigo recebido em 08/07/2019, revisado em 23/03/2020, aceito em 14/05/2020

DOI: https://doi.org/10.36660/abc.20190089 


\begin{abstract}
$3.42 \pm 1.92$, which is similar to that of non-octogenarians $(3.44 \pm 1.74 ; p=0.92$ in univariate analysis and $p=0.80$ after adjustment for sex by analysis of variance - ANOVA). There was also no statistically significant difference when the sample was divided into median age (62 years; $3.41 \pm 1.77$ vs. $3.49 \pm 1.77 ; p=0.61)$. There was no statistically significant linear association between age and typicality index ( $r=-0.05$; $p=0.24)$. Logistic regression analysis for prediction of CAD in the general sample of 958 patients showed no interaction of typicality index with numeric age $(p=0.94)$, octogenarians $(p=0.22)$ or age above median $(p=0.74)$.
\end{abstract}

Conclusion: In patients with acute chest pain of coronary etiology, advanced age does not influence the typical clinical presentation.

Keywords: Elderly; Chest Pain; Acute Coronary Syndrome; Prognosis.

Full texts in English - http://www.arquivosonline.com.br

\section{Introdução}

O pensamento clínico tradicional indica que os idosos estão predispostos a sintomas atípicos nas síndromes coronarianas agudas (SCA), condição que pode implicar em difícil diagnóstico e tratamento tardio. ${ }^{1}$ Os mecanismos plausíveis para atipicidade seriam limitações cognitivas, comunicação comprometida ou redução da percepção da dor. ${ }^{2}$

No entanto, embora esse pensamento clínico tradicional tenha como possível base fisiológica a alteração nociceptiva causada pela depressão e diabetes, que são mais prevalentes em indivíduos com mais idade, a grande maioria dos estudos encontrados na literatura é retrospectiva e com uma definição bastante variável e subjetiva de "tipicidade da dor". Portanto, ainda não está claro se, de fato, a idade avançada implica um quadro clínico diferente no contexto das síndromes coronarianas. ${ }^{3,4}$

Assim, o presente estudo se propõe a testar a hipótese de que indivíduos muito idosos estão mais predispostos a manifestações atípicas de dor torácica de etiologia coronariana. Como uma análise primária, a tipicidade geral da manifestação clínica foi comparada entre octogenários e não octogenários na subamostra de pacientes com etiologia coronariana. Seguiu-se a análise da interação entre idade e tipicidade da dor na predição da etiologia coronariana na amostra de todas as etiologias de dor torácica.

\section{Métodos}

\section{Seleção da amostra}

O Registro de Dor Torácica é uma amostra de pacientes internados consecutivamente na Unidade Coronariana de um hospital terciário de setembro de 2011 a dezembro de 2017, principalmente por desconforto torácico, independentemente de alterações eletrocardiográficas, marcadores de necrose ou qualquer outro exame complementar que mostre a causa do sintoma.

A amostra selecionada tem como objetivo representar a população-alvo de pacientes internados na unidade coronariana devido a dor torácica. Assim, todos os pacientes internados durante o período do estudo foram incluídos no estudo, não havendo seleção de subamostra nesta população. A internação na unidade coronariana não foi influenciada pelo protocolo do estudo. A probabilidade diagnóstica foi estabelecida a critério dos médicos assistentes.

O estudo está de acordo com as normas éticas da resolução 510/2016 do Ministério da Saúde, foi aprovado pelo Comitê de Ética em Pesquisa Hospitalar e todos os participantes assinaram o termo de consentimento livre e esclarecido.

\section{Caracterização do desconforto torácico}

$\mathrm{Na}$ internação, as informações sobre a apresentação clínica do desconforto torácico foram coletadas por meio de entrevista parametrizada. A entrevista foi realizada de forma sistematizada por pesquisadores treinados para evitar induzir as respostas dos pacientes e focar na reprodutibilidade do método. A entrevista foi parametrizada para exigir respostas objetivas sim/não. Quando o paciente manifestou dúvida, o sintoma foi considerado ausente.

Foram avaliadas 12 características de sintomas, incluindo 8 características consideradas típicas de angina (dor precordial, aspecto compressivo, irradiação para membro superior esquerdo, irradiação para o pescoço, intensidade classificada pelo paciente como grave, desconforto nos dias anteriores, presença de sintomas vagais, administração de medicação sublingual seguida de melhora do sintoma) e 4 características consideradas atípicas (mudança da dor conforme a posição, mudança com palpação do local, mudança com movimento do braço e mudança com respiração).

\section{Índice de tipicidade de sintomas}

Para quantificar a tipicidade geral da manifestação clínica, atribuiu-se 1 ponto a cada característica típica e subtraiu-se 1 ponto para cada característica atípica (variação de -4 a +8 , proporcional à tipicidade).

\section{Definição da etiologia dos sintomas}

Para a avaliação diagnóstica, os pacientes foram submetidos a angiografia coronária invasiva ou teste provocativo não invasivo (ressonância magnética de perfusão nuclear e tomografia computadorizada por emissão de fóton único ou ecocardiografia sob estresse com dobutamina), a critério do cardiologista responsável. Para testes não invasivos positivos, os pacientes fizeram uma angiografia para confirmação. Com base nesse algoritmo de diagnóstico, definiu-se doença arterial coronariana obstrutiva (DACO) como estenose $\geq 70 \%$ na angiografia. A angiografia coronária sem lesão obstrutiva ou teste não invasivo normal (tamanho do defeito isquêmico $<5 \%$ do miocárdio ventricular esquerdo) indicou ausência de DACO.

\section{Análise dos dados}

A normalidade das variáveis numéricas foi testada por histograma, comparando média e mediana, e considerando principalmente o nível de curtose e assimetria $<3$. Indivíduos muito idosos foram definidos com idade $\geq 80$ anos (octogenários). Realizou-se a análise preliminar na amostra de pacientes com doença coronariana obstrutiva, comparando o 
índice de tipicidade entre octogenários e não octogenários. Além disso, comparou-se cada característica de sintomas entre os dois grupos. As variáveis numéricas foram expressas como média e desvio padrão, comparadas entre os dois grupos pelo teste t de Student não pareado. As variáveis categóricas foram expressas em proporções e comparadas com o teste qui-quadrado de Pearson. Procedeu-se à análise de variância para comparar o índice de tipicidade entre os grupos após ajuste para sexo. A associação linear entre índice de tipicidade e idade foi testada pelo coeficiente de correlação de Pearson, com base na distribuição normal de ambas as variáveis. A comparação múltipla foi ajustada pelo método de Bonferroni.

Em seguida, utilizou-se a amostra total do registro (todos os pacientes admitidos com dor torácica aguda, com e sem doença arterial coronariana), e avaliamos a capacidade preditiva do índice de tipicidade para doença arterial coronariana obstrutiva com base na área sob a curva Receiver Operator Characteristic (ROC). Depois, avaliamos o efeito modificador da idade sobre a precisão do diagnóstico (DACO) da tipicidade geral da dor, em termos de interação vs. tipicidade da idade na regressão logística, com a idade sendo inserida de três maneiras diferentes: como uma variável numérica, categorizada em dois grupos (octogenários ou não octogenários) e categorizada em dois grupos a partir da mediana da amostra. Para a análise estatística, foi utilizado o software SPSS versão 23. A significância estatística foi definida como um valor de p bicaudal menor que 0,05.

\section{Cálculo amostral}

Quanto ao cálculo amostral, trata-se de um estudo realizado em amostra previamente existente no Registro de Dor Torácica, uma coleta prospectiva de pacientes internados por dor torácica. Esse registro é utilizado para várias análises e, em nossa metodologia, antes de decidirmos testar qualquer hipótese, avaliamos o poder estatístico, que depende do comportamento da variável em questão. Assim, como os dados já haviam sido coletados, poderíamos utilizar o desvio padrão da amostra que seria utilizado para avaliar se o tamanho da amostra tinha poder suficiente, critério essencial para permitir a análise dos dados em nosso protocolo.

Assim, o tamanho da amostra foi definido primeiro, com base na distribuição do índice de tipicidade na amostra de doença coronariana. Considerando o desvio padrão de 1,7, seria necessário que 36 octogenários e 109 não octogenários oferecessem $80 \%$ de poder na detecção de uma diferença de 30\% no índice típico pelo teste t de Student.

\section{Resultados}

\section{Caracterização da amostra}

Entre setembro de 2011 e dezembro de 2017, 958 indivíduos foram incluídos no registro, e 486 (51\%) tinham etiologia supostamente coronariana. Nesse grupo, 59 octogenários foram comparados a 427 não octogenários. A média de idade dos octogenários foi de 85 23,4 anos, sendo $56 \%$ homens, em comparação com $60 \pm 12$ anos, sendo $71 \%$ homens, no grupo de não octogenários $(p<0,001)$. Pacientes octogenários tiveram uma prevalência maior de disfunção ventricular esquerda clinicamente manifesta ( $24 \%$ versus $8,7 \%$, $\mathrm{p}<0,001$ ), doença triarterial ou tronco de coronária esquerda ( $41 \%$ versus $26 \%, p=0,01$ ) e menor prevalência de infarto agudo do miocárdio com elevação do segmento ST (25\% versus $30 \%$, $\mathrm{p}<0,001)$. A mortalidade foi maior no grupo com idade mais avançada (14\% versus $2,1 \%, p<0,001)$. As variáveis comparadas entre os dois grupos encontram-se discriminadas na tabela 1.

\section{Idade e tipicidade dos sintomas}

O índice de tipicidade dos pacientes muito idosos foi de $3,42 \pm 1,92$, semelhante ao observado em indivíduos mais

Tabela 1 - Características clínicas e comorbidades

\begin{tabular}{|c|c|c|c|}
\hline & $\begin{array}{c}\text { Idade }<80 \text { anos } \\
(n=427)\end{array}$ & $\begin{array}{c}\text { Idade } \geq 80 \text { anos } \\
\quad(n=59)\end{array}$ & Valor de $p$ \\
\hline Idade (anos) & $60 \pm 12$ & $85 \pm 3,4$ & $<0,001$ \\
\hline Homens & $302(71 \%)$ & $33(56 \%)$ & 0,02 \\
\hline Isquemia ao ECG & $279(67 \%)$ & $37(65 \%)$ & 0,80 \\
\hline Troponina positiva & $274(65 \%)$ & $49(83 \%)$ & 0,005 \\
\hline Infarto com supradesnivelamento do segmento ST & $127(30 \%)$ & $15(25 \%)$ & $<0,001$ \\
\hline Diabetes mellitus & $161(38 \%)$ & $27(46 \%)$ & 0,23 \\
\hline Creatinina $(\mathrm{mg} / \mathrm{dL})$ & $1,0 \pm 0,69$ & $1,1 \pm 0,43$ & 0,12 \\
\hline Pressão arterial sistólica (mmHg) & $154 \pm 31$ & $153 \pm 36$ & 0,08 \\
\hline Frequência cardíaca (bpm) & $78 \pm 18$ & $76 \pm 19$ & 0,17 \\
\hline Doença coronariana prévia & $139(33 \%)$ & $24(41 \%)$ & 0,22 \\
\hline Revascularização miocárdica prévia & $37(8,7 \%)$ & $7(12 \%)$ & 0,40 \\
\hline Padrão anatômico grave* & $80(26 \%)$ & $16(41 \%)$ & 0,01 \\
\hline
\end{tabular}

*Cateterismo com obstrução $\geq 70 \%$. ECG: eletrocardiograma. 
jovens $(3,44 \pm 1,74 ; p=0,92)$. A comparação do índice de tipicidade permaneceu não significativa $(p=0,80)$ após ajuste para diferença de gênero entre os grupos (Figura 1).

Não houve diferença no índice de tipicidade quando a amostra foi dividida em idade mediana (62 anos), sendo $3,41 \pm 1,77$ versus $3,49 \pm 1,77(p=0,61)$. Da mesma forma, não houve correlação entre índice típico e idade ( $r=-$ $0,05, p=0,24)$ (Figura 2).

A comparação entre as 12 características da dor entre octogenários e não octogenários não mostrou diferença significativa após o ajuste de Bonferroni (Tabela 2).

\section{Efeito modificador da idade na capacidade preditiva da tipicidade da dor}

Analisando os 958 pacientes do registro, o índice de tipicidade apresentou uma área sob a curva ROC de 0,62 (IC 95\% = 0,58-0,65) para predição de doença arterial coronariana obstrutiva. A análise de regressão logística não demonstrou interação do índice de tipicidade com idade numérica $(p=0,94)$, octogenários $(p=0,22)$ ou idade acima da mediana de 62 anos $(p=0,74)$ (Figura 3).

\section{Discussão}

O presente estudo demonstra que a idade avançada não influencia a tipicidade da apresentação clínica no contexto das síndromes coronarianas agudas. Além disso, o valor diagnóstico da manifestação clínica não é influenciado pela idade. Conforme mostrado, mesmo analisando a "idade avançada" sob várias perspectivas (dividindo a amostra entre octogenários e não octogenários, idade mediana, 62 anos, e ainda colocando a idade como uma variável contínua), nenhuma das análises sugeriu influência.

A utilização de um "índice de tipicidade" permitiu analisar a tipologia geral da dor, informação complementada pela análise individual de cada característica. O chamado "índice" é apenas a contagem dos sintomas sugeridos menos os sintomas não sugeridos, uma forma de tratar a tipicidade como variável numérica, evitando a subjetividade da categorização em um quadro clínico típico ou atípico.

Outro ponto importante deste estudo é que, para a definição de DACO, utilizamos a cinecoronariografia, exame padrão-ouro, o que implica baixo risco de viés de calibração.

Estudos anteriores que buscaram estudar a dor em indivíduos com idade mais avançada com síndrome coronariana aguda mostraram resultados controversos. . $^{2,3}$ Verifica-se que na maioria desses estudos a coleta das características da dor foi feita retrospectivamente e a partir de bancos de dados desenvolvidos com outros objetivos primários.

Em 2001, Mehta et al.,' por meio de um registro de beneficiários do Medicare nos EUA, selecionaram pacientes com diagnóstico de infarto agudo do miocárdio e estratificaram a amostra com base na idade. ${ }^{1}$ Em seu estudo, os autores concluem que a apresentação inicial

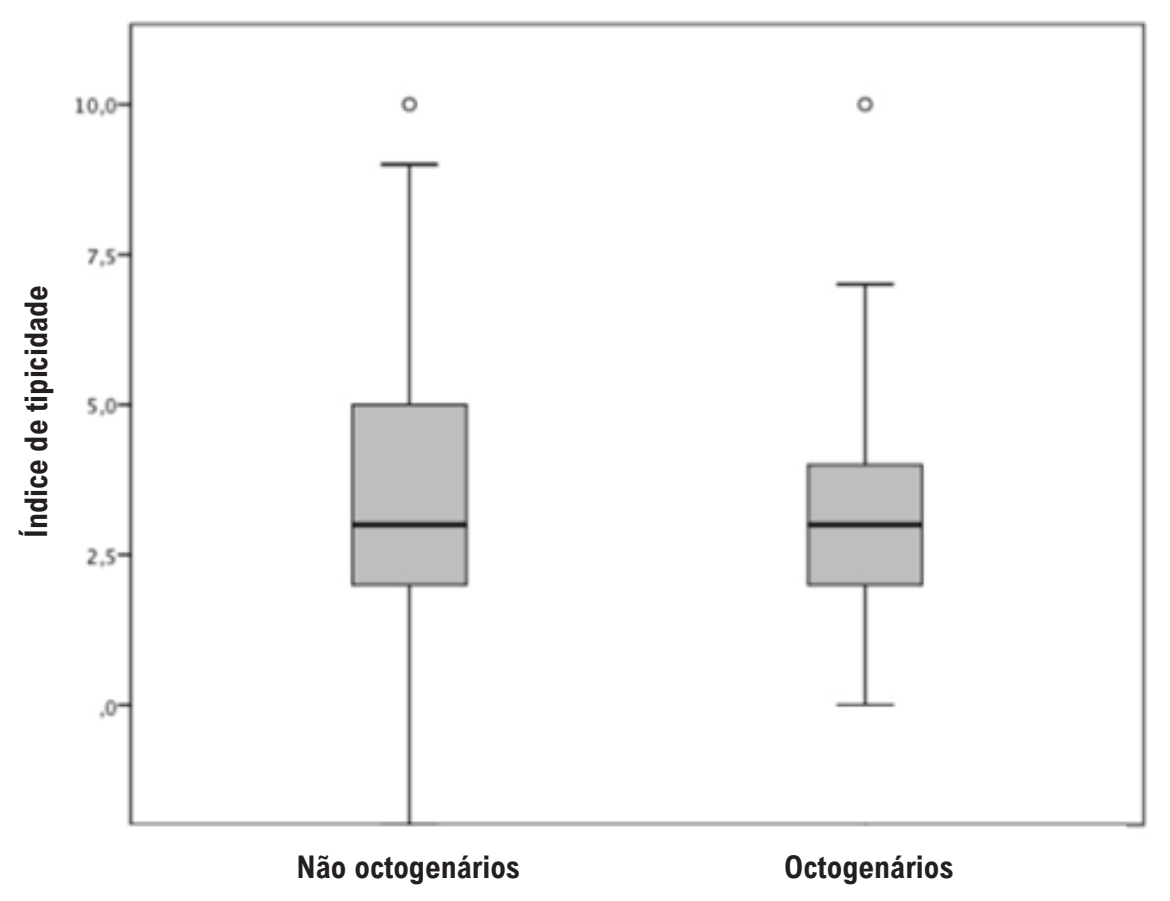

Figura 1 - Boxplot do índice de tipicidade para os grupos octogenários e não octogenários $(p=0,92)$. 


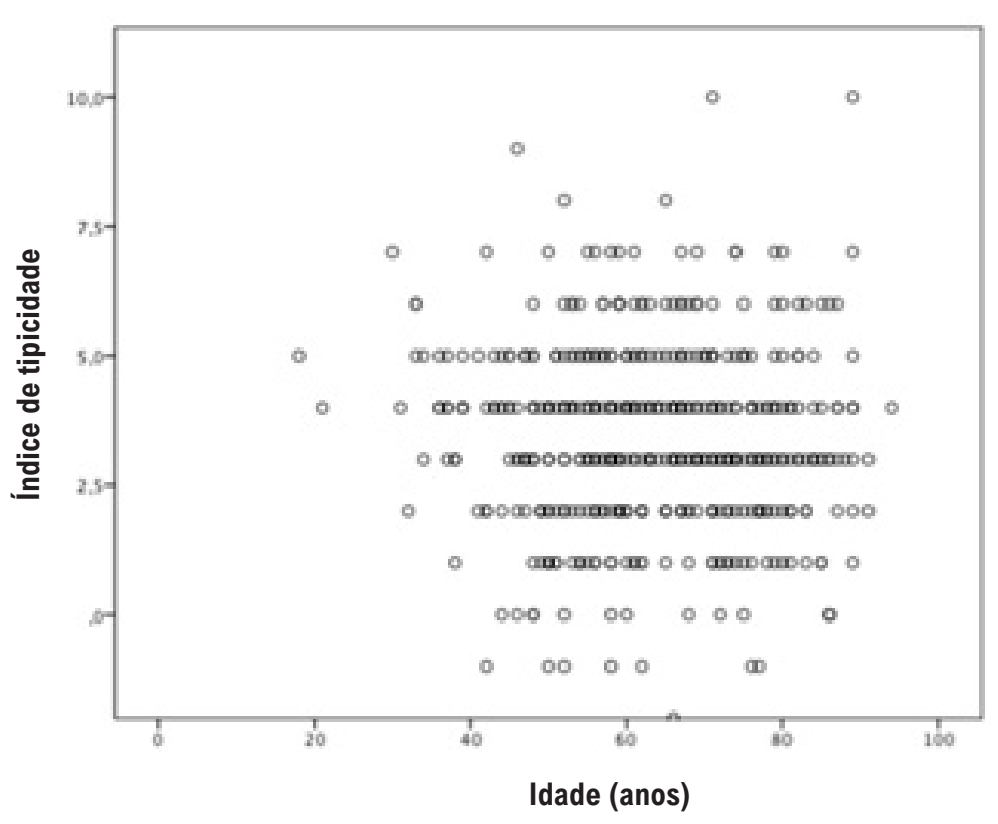

Figura 2 - Gráfico de dispersão do índice típico e idade.

Tabela 2 - Características da dor torácica

\begin{tabular}{lcccc}
\hline & Idade $\geq \mathbf{8 0}$ anos & Idade $<80$ anos & Valor de p & $\begin{array}{c}\text { Valor de } \mathbf{p} \text { ajustado } \\
\text { (Bonferroni) }\end{array}$ \\
\hline Dor precordial & $348(82 \%)$ & $48(81 \%)$ & 0,98 & -- \\
\hline Dor compressiva & $241(56 \%)$ & $28(48 \%)$ & 0,19 & -- \\
\hline Irradiação para o membro superior esquerdo & $167(39 \%)$ & $19(32 \%)$ & 0,02 & 0,24 \\
\hline Irradiação para o pescoço & $110(26 \%)$ & $8(14 \%)$ & 0,04 & 0,48 \\
\hline Intensidade severa & $253(60 \%)$ & $39(66 \%)$ & 0,33 & -- \\
\hline Desconforto nos dias anteriores & $67(48 \%)$ & $14(67 \%)$ & 0,10 & -- \\
\hline Sintomas vagais & $215(50 \%)$ & $32(54 \%)$ & 0,58 & -- \\
\hline Melhora com nitrato & $182(43 \%)$ & $18(31 \%)$ & 0,08 & -- \\
\hline Muda com a posição & $70(16 \%)$ & $7(12 \%)$ & 0,37 & -- \\
\hline Muda com palpação & $26(6,1 \%)$ & $2(3,4 \%)$ & 0,40 & -- \\
\hline Muda com o movimento do braço & $29(6,8 \%)$ & $3(5,1 \%)$ & 0,62 & -- \\
\hline Dor pleurítica & $51(12 \%)$ & $5(8,5 \%)$ & 0,43 & \\
\hline
\end{tabular}

da dor torácica diminui com o aumento da idade. No entanto, não mostram se há diferença estatística entre os valores, o que torna essa conclusão enganosa.

Em uma análise post-hoc do registro Internet Tracking Registry for Acute Coronary Syndromes (i*trACS), Han et al., ${ }^{3}$ analisaram a apresentação clínica em pacientes com SCA de dois grupos: idade $\geq 75$ anos e $<75$ anos. Classificaram a "apresentação típica" como dor torácica em esmagamento, compressão ou pressão e concluíram que apenas no grupo de pacientes mais jovens (idade $<75$ anos) a apresentação típica estava associada ao diagnóstico de SCA. Além da definição simplista de "apresentação típica", os autores não compararam as duas faixas etárias com diagnóstico de SCA. Em outra análise post-hoc, do Gulf Registry of Acute Coronary Events (Gulf RACE), ElMenyar et al. ${ }^{5}$ classificaram em 3 categorias: típica, atípica e dispneia, não sendo encontrada diferença de idade nos grupos de apresentação "típica" (55 \pm 12$)$ e "atípica" $(57 \pm 13)$. No entanto, os autores atribuem características bastante amplas como sendo "típicas": "irradiação para o braço, ombro, costas, pescoço, mandíbula, epigástrio ou outros locais," o que torna essa classificação subjetiva. 


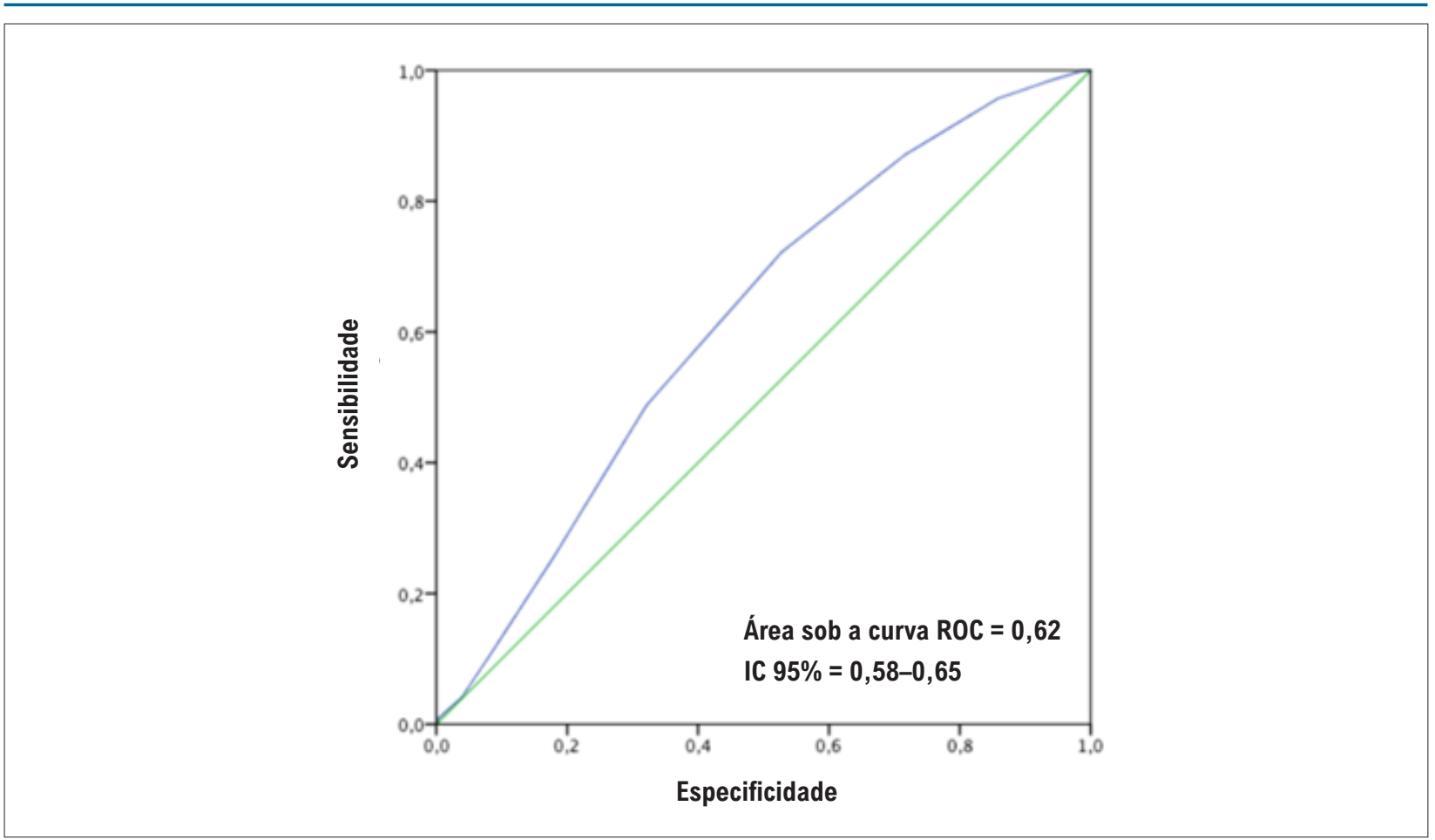

Figura 3 - Estatística C do índice de tipicidade para predição de doença arterial coronariana obstrutiva, considerando todos os pacientes no registro (958), curva ROC: 0,62 $(95 \% \mathrm{Cl}=0,58-0,65)$.

Devemos reconhecer que, apesar de satisfazer o cálculo amostral, nossa população de pacientes muito idosos era pequena. Além disso, nosso estudo foi realizado em apenas um centro e em uma população selecionada, sendo necessário o desenvolvimento de novos estudos neste contexto. Reconhecemos também que este estudo foi realizado em ambiente de hospital terciário, portanto, devemos ter cuidado ao extrapolar seus resultados para o ambiente de atenção primária. Nossa população de maior interesse é a de pacientes internados em unidade coronariana, população em que o desafio da discriminação diagnóstica é maior, pois há maior homogeneidade de sintomas (zona de probabilidade cinza). Por ser nossa população-alvo, não houve viés de seleção. Finalmente, existe uma infinidade de possibilidades e combinações de sintomas a serem incluídos em uma análise como esta. Mas aqui, não estamos tentando criar um escore preditor para a etiologia da dor; estamos apenas comparando os muito idosos com os não muito idosos quanto à "carga de tipicidade". Independentemente de contemplarmos todos os sintomas possíveis, o teste de hipótese para a "carga de tipicidade" não fica comprometido. Estamos apenas avaliando se existe um gradiente de sintomas entre esses dois grupos.

\section{Conclusão}

Em pacientes com dor torácica de etiologia coronariana, a idade avançada não parece influenciar a apresentação clínica típica, sugerindo que os sintomas devam ser interpretados independentemente da idade.

\section{Contribuição dos autores}

Concepção e desenho da pesquisa: Filgueiras PHC, Cerqueira Junior AM, Correia LCL, Lopes FOA; Obtenção de dados: Filgueiras PHC, Cerqueira Junior AM, Bagano GO, Correia VCA, Souza TMB, Fonseca LL, Kertzman LQ, Lacerda YF, Rabelo MN, Lopes FOA; Análise e interpretação dos dados: Filgueiras PHC, Cerqueira Junior AM, Bagano GO, Correia VCA, Souza TMB, Lopes FOA; Análise estatística: Filgueiras PHC, Cerqueira Junior AM, Bagano GO, Correia VCA, Fonseca LL, Kertzman LQ, Lacerda YF, Rabelo MN, Correia LCL, Lopes FOA; Revisão crítica do manuscrito quanto ao conteúdo intelectual importante: Filgueiras PHC, Cerqueira Junior AM, Bagano GO, Correia VCA, Souza TMB, Fonseca LL, Kertzman LQ, Lacerda YF, Rabelo MN, Correia LCL, Lopes FOA.

\section{Potencial conflito de interesse}

Não há conflito com o presente artigo

\section{Fontes de financiamento}

O presente estudo não teve fontes de financiamento externas.

\section{Vinculação acadêmica}

Este artigo é parte de tese de doutorado de Antônio Maurício Cerqueira Junior pela Escola Bahiana de Medicina e Saúde Pública. 


\section{Referências}

1. Mehta RH, Rathore SS, Radford MJ, Wang Y, Krumholz HM. Acute myocardial infarction in the elderly: differences by age. J Am Coll Cardiol. 2001;38(3):736-41.

2. Grosmaitre P, Le Vavasseur O, Yachouh E, Courtial Y, Jacob X, Meyran S, et al. Significance of atypical symptoms for the diagnosis and management of myocardial infarction in elderly patients admitted to emergency departments. Arch Cardiovasc Dis. 2013;106(11):586-92.

3. Han JH, Lindsell CJ, Hornung RW, Lewis T, Storrow AB, Hoekstra JW, et al. The elder patient with suspected acute coronary syndromes in the emergency department. Acad Emerg Med. 2007;14(8):732-9.
4. Goch A, Misiewicz P, Rysz J, Banach M. The clinical manifestation of myocardial infarction in elderly patients. Clin Cardiol. 2009;32(6). E46-51.

5. El-Menyar A, Zubaid M, Shehab A, Bulbanat B, Albustani N, Alenezi F, et al. Prevalence and impact of cardiovascular risk factors among patients presenting with acute coronary syndrome in the middle east. Clin Cardiol. 2011;34(1):51-8. 